\title{
MORDELL'S EQUATION IN CHARACTERISTIC THREE
}

\author{
J.F. VOLOCH
}

Let $K$ be a function field in one variable over a finite field of characteristic three. If $a \in K$ is not a cube, we show that the equation $y^{2}=x^{3}+a$ has only finitely many solutions $x, y \in K$.

In this note we will study the equation $y^{2}=x^{3}+a, \quad a \in K$, where $K$ is a function field in one variable over the perfect field $k$, of characteristic 3 .

The analogous equation over $\mathbf{Q}$ is called Mordell's equation and has been extensively studied by Mordell and others $([2])$. Over a field of characteristic not equal to 2 or 3 , the equation $y^{2}=x^{3}+a, \quad a \neq 0$ defines an elliptic curve. In these cases the general theory of elliptic curves can be brought to bear on the problem ([4]).

In characteristic 3 , the equation $y^{2}=x^{3}+a$ defines a rational curve over $\bar{K}$ but, if $a$ is not a cube, it is a curve of genus 1 over $K$ (under the definition of genus of [1], for example). When $a=b^{3}$ then the change of variables $x=x_{1}-b$ takes the equation to $y^{2}=x_{1}^{3}$ which has as general solution $x_{1}=t^{2}, \quad y=t^{3}, \quad t \in K$. When $a \notin K^{3}$ the result is surprisingly different. The following result is a corollary of the theorem below.

Corollary. If $k$ is a finite field and $a \in K \backslash K^{3}$ then $y^{2}=x^{3}+a$ has only finitely many solutions $x, y \in \dot{K}$.

Before stating our theorem let is introduce some notation. Let $C$ be the curve $y^{2}=x^{3}+a, \quad a \in K \backslash K^{3}$ and denote by $C(L)$ the $L$-rational points of $C$ for $L \supset K$, any field. Since $C$ is a cubic we can define a group law on the nonsingular (projective) points of $C(\bar{K})$ by the usual chord and tangent method. The singular point of $C$ is $\left(-a^{1 / 3}, 0\right)$ which is not on $C(K)$, thus $C(K)$ is a group with identity $\mathcal{O}$, the point at infinity. Finally, since $a \notin K^{3}$ we can consider the derivation $d / d a$ of $K$.

Theorem. Let $\mu: C(K) \rightarrow K^{+}$be defined by $\mu(\mathcal{O})=0, \mu((x, y))=d y / d a$. Then $\mu$ is an injective homomorphism. Further, there exists a divisor $D$ of $K$ with $\mu(C(K)) \subseteq L(D)$.

Proof: Let $P_{i}=\left(x_{i}, y_{i}\right) \in C(K), \quad i=1,2,3$, satisfy $P_{1}+P_{2}+P_{3}=0$. Then $P_{1}, P_{2}, P_{3}$ are collinear, hence there exists $\alpha, \beta \in K, y_{i}=\alpha x_{i}+\beta, i=1,2,3$.

Received 30th March 1989

Copyright Clearance Centre, Inc. Serial-fee code: 0004-9729/90 \$A2.00+0.00. 
Therefore $x_{1}, x_{2}, x_{3}$ satisfy the equation $x^{3}+a-(\alpha x+\beta)^{2}=0$, so $x_{1}+x_{2}+x_{3}=\alpha^{2}$. It follows that

$$
y_{1}+y_{2}+y_{3}=\alpha\left(x_{1}+x_{2}+x_{3}\right)+3 \beta=\alpha^{3} .
$$

Whence, applying $d / d a$,

$$
\mu\left(P_{1}\right)+\mu\left(P_{2}\right)+\mu\left(P_{3}\right)=0 .
$$

It follows that $\mu$ is a homomorphism.

Differentiating the defining equation $y^{2}=x^{3}+a$, we get $2 y d y / d a=1$, so $\mu(P) \neq 0$ for $P \neq \mathcal{O}$, and $\mu$ is injective.

Now, let $v$ be a place of $K$ and $P=(x, y) \in C(K)$. As $v(d y) \geqslant v(y)-1$, we have $v(y) \leqslant v(\mu(P))+v(d a)+1$. On the other hand $2 y d y / d a=1$, so

$$
\begin{aligned}
& v(\mu(P))=-v(y) \geqslant-(v(\mu(P))+v(d a)+1) . \\
& \text { It follows that } \quad v(\mu(P)) \geqslant-\frac{(v(d a)+1)}{2} \text {. }
\end{aligned}
$$

If $v(d a)=0$, this of course improves to $v(\mu(P)) \geqslant 0$ and the result follows.

REMARKs. (1) A similar behaviour to that observed above in characteristic 3 happens in characteristic 2 with Mordell's equation. More generally the equation $y^{2}=x^{3}+a x+b$, where $a$ or $b$ is not a square, is the most general curve in characteristic 2 of nonconservative genus 1 , possessing a rational point. In fact this curve and Mordell's equation in characteristic 3 are the only such curves ([3]). We have also analogous results in this case taking $\mu(x, y)=d x / d t$ for some $t \in K \backslash K^{2}$. The theorem and its corollary hold in this case as well except for the fact that $\operatorname{ker} \mu=\{\mathcal{O},(u, v)\}$, where $u=d b / d a$, if $a \notin K^{2}$. We leave the details to the reader.

(2) The map $\mu$ constructed above may seem rather mysterious but it is the analogue, in this situation, of Manin's map. (See [5]).

\section{REFERENCES}

[1] C. Chevalley, Introduction to the Theory of algebraic functions of one variable (American Mathematical Society, New York, 1951).

[2] L.J. Mordell, Diophantine Equations (Academic Press, New York, 1969).

[3] C.S. Queen, 'Non-conservative function fields of genus one, I, II', Arch. Math. 22 (612-623). and 23 (1972) pp. 30-37.

[4] J.H. Silverman, The Arithmetic of elliptic curves (Springer, New York, 1986).

[5] J.F. Voloch, 'Explicit p-descent for elliptic curves in characteristic p', Compositio Math. (to appear).

IMPA

Estrada D. Castorina 110

llio de Janeiro 22460 Brasil 